\title{
Reduction in Biogenic Amine Content in Baechu (Napa Cabbage) Kimchi by Biogenic Amine-Degrading Lactic Acid Bacteria
}

\author{
Junsu Lee, Young Hun Jin (D), Alixander Mattay Pawluk (D) and Jae-Hyung Mah *(D) \\ Department of Food and Biotechnology, Korea University, 2511 Sejong-ro, Sejong 30019, Korea \\ jpang@korea.ac.kr (J.L.); younghoonjin3090@korea.ac.kr (Y.H.J.); alixei@korea.ac.kr (A.M.P.) \\ * Correspondence: nextbio@korea.ac.kr; Tel.: +82-44-860-1431
}

Citation: Lee, J.; Jin, Y.H.; Pawluk, A.M.; Mah, J.-H. Reduction in Biogenic Amine Content in Baechu (Napa Cabbage) Kimchi by Biogenic Amine-Degrading Lactic Acid Bacteria. Microorganisms 2021, 9, 2570. https://doi.org/10.3390/

microorganisms 9122570

Academic Editor: Franca Rossi

Received: 18 November 2021 Accepted: 10 December 2021 Published: 13 December 2021

Publisher's Note: MDPI stays neutral with regard to jurisdictional claims in published maps and institutional affiliations.

Copyright: (c) 2021 by the authors. Licensee MDPI, Basel, Switzerland. This article is an open access article distributed under the terms and conditions of the Creative Commons Attribution (CC BY) license (https:// creativecommons.org/licenses/by/ $4.0 /)$.

\begin{abstract}
This study was performed to mine biogenic amine (BA)-degrading lactic acid bacteria (LAB) from kimchi and to investigate the effects of the LAB strains on BA reduction in Baechu kimchi fermentation. Among $1448 \mathrm{LAB}$ strains isolated from various kimchi varieties, five strains capable of considerably degrading histamine and/or tyramine were selected through in vitro tests and identified as Levilactobacillus brevis PK08, Lactiplantibacillus pentosus PK05, Leuconostoc mesenteroides YM20, L. plantarum KD15, and Latilactobacillus sakei YM21. The selected strains were used to ferment five groups of Baechu kimchi, respectively. The LB group inoculated with L. brevis PK08 showed the highest reduction in tyramine content, $66.65 \%$ and $81.89 \%$, compared to the control group and the positive control group, respectively. Other BA content was also considerably reduced, by $3.76-89.26 \%$ (five BAs) and 7.87-23.27\% (four BAs), compared to the two control groups, respectively. The other inoculated groups showed similar or less BA reduction than the LB group. Meanwhile, a multicopper oxidase gene was detected in L. brevis PK08 when pursuing the BA degradation mechanism. Consequently, L. brevis PK08 could be applied to kimchi fermentation as a starter or protective culture to improve the BA-related safety of kimchi where prolific tyramine-producing LAB strains are present.
\end{abstract}

Keywords: biogenic amine; lactic acid bacteria; biogenic amine degradation; multicopper oxidase; Baechu kimchi; fermentation

\section{Introduction}

Biogenic amines (BAs), such as tyramine, histamine, putrescine, cadaverine, spermidine, spermine, tryptamine, and $\beta$-phenylethylamine, are basic nitrogenous organic compounds that are produced mainly though microbial decarboxylation of precursor amino acids [1]. Although the BAs are generally vital for biological functions such as the regulation of the nervous system, the cardiovascular system, and body temperature, the excessive consumption of foods with high levels of BAs, as well as inhibition and deficiency of amine oxidase by specific inhibitors, occasionally induces several adverse health effects [2]. Among BAs, tyramine and histamine (toxic BAs) are considered to be the most toxic due to numerous toxicological effects [2,3]. The over-consumption of foods containing high levels of these BAs may potentially cause histamine poisoning (e.g., scombroid poisoning) or tyramine toxicity (e.g., cheese crisis) with various symptoms including headache, nausea, respiratory difficulties, migraine, vomiting, brain haemorrhage, heart failure, hypoor hypertension, etc. [4,5]. Additionally, polyamines, including cadaverine, putrescine, spermine, and spermidine, have the potential to interrupt the metabolization of tyramine and histamine, in turn, enhancing their toxicity, and may possibly cause the formation of carcinogenic N-nitrosamines by reacting with nitrite [6,7]. According to the EFSA [3], an intake of $50 \mathrm{mg}$ histamine or $600 \mathrm{mg}$ tyramine per meal showed no adverse effects for healthy individuals, while these concentrations were much lower for individuals taking 
monoamine oxidase inhibitors. Several studies have suggested $<100 \mathrm{mg} / \mathrm{kg}$ of histamine, $<100$ to $800 \mathrm{mg} / \mathrm{kg}$ of tyramine, $<30 \mathrm{mg} / \mathrm{kg}$ of $\beta$-phenylethylamine, and $<1000 \mathrm{mg} / \mathrm{kg}$ of total BAs as the safety criteria (upper limits) for BAs in food $[1,8]$. Although toxicity limits of BAs have been studied [9-13], there are no regulations, except for histamine, set by governments and international organizations. Even more limiting, current regulations by the European Commission (EC) and the U.S. Food and Drug Administration (FDA) are in place only in regard to the histamine content of 50 to $100 \mathrm{mg} / \mathrm{kg}$ in fish and $400 \mathrm{mg} / \mathrm{kg}$ in fish sauces and other fish products $[14,15]$.

The term kimchi describes a wide variety of traditional Korean lactic acid-fermented vegetable products that are commonly produced through natural fermentation without the use of a starter culture. Baechu kimchi (napa cabbage kimchi), in particular, is prepared by mixing salted napa cabbage with seasoning ingredients composed of radish, garlic, ginger, green onion, and red pepper powder, followed by fermentation at low temperatures (typically at 2 to $10^{\circ} \mathrm{C}$ ) for a few weeks $[16,17]$. Other seasoning ingredients such as Jeotgal and Aekjeot (salted and fermented seafood products) are also frequently added [18]. It is known that the microflora of kimchi is composed mainly of lactic acid bacteria (LAB) species, including Lactobacillus, Leuconostoc, and Weissella spp., which are the major microorganisms responsible for kimchi fermentation $[19,20]$. The microflora of kimchi varieties differs considerably depending on the main and seasoning ingredients used and the fermentation conditions, as well as physicochemical and biological factors in preparing the kimchi [16]. In the processes of kimchi fermentation, diverse LAB derived from the raw ingredients play a vital role in producing vitamins, organic acids, carbon dioxide, ethanol, bacteriocins, and prebiotic factors, which affect the organoleptic properties of the final kimchi product $[21,22]$. Several studies have reported on the numerous beneficial properties and functionalities of kimchi, including anti-mutagenic [23], anti-diabetic [24], anti-obese [25], anti-aging [26], antioxidative [27], and anti-carcinogenic effects [28]. Despite the numerous benefits of kimchi, potentially hazardous substances, such as BAs, may be formed by microbial enzymatic activity and/or by undesirable influences of the ingredients used in kimchi preparation [29,30]. There are numerous reports [31-35] on tyramine and/or histamine concentrations in different types of kimchi, including Beach $u$ kimchi, that were higher than the upper limits suggested by Ten Brink et al. [8]. For instance, several studies have described commercial Baechu kimchi as containing histamine up to $142.3 \mathrm{mg} / \mathrm{kg}$ and tyramine up to $118.2 \mathrm{mg} / \mathrm{kg}[31,33]$. Surprisingly, another previous study reported commercial Baechu kimchi to contain histamine up to $5350 \mathrm{mg} / \mathrm{kg}$ [35]. Histamine contents of up to $386.03 \mathrm{mg} / \mathrm{kg}$ and tyramine contents of up to $181.10 \mathrm{mg} / \mathrm{kg}$ in other types of kimchi, including Kkakdugi (kimchi made mainly of diced radish), Chonggak kimchi (kimchi made mainly of ponytail radish), $\mathrm{Pa}$ kimchi (kimchi made mainly of green onion), and Gat kimchi (kimchi made mainly of mustard leaf), have also been reported in previous studies [32,34].

The majority of solutions for reducing BAs in foods have been well reviewed in literature, and include additives, irradiation, packaging, antimicrobial compounds, starter cultures, and control or adjustment of environmental factors [36,37]. Particularly, in fermented foods, utilizing microorganisms capable of degrading and/or incapable of producing BAs as starter cultures is considered to be one of the most promising strategies, because they provide less or no unfavorable organoleptic and unhealthy alterations and insignificant changes in microbial communities in the foods compared to other treatments [30,37]. Diverse $\mathrm{LAB}$ species that have been studied to degrade BAs in fermented foods include: Companilactobacillus farciminis (formerly Lactobacillus farciminis), Lactiplantibacillus plantarum (formerly Lactobacillus plantarum), and Pediococcus acidilactici for histamine, tyramine, and putrescine reduction in wine [38]; Latilactobacillus sakei (formerly Lactobacillus sakei) for histamine reduction in fish slurry [39]; L. plantarum for tyramine, putrescine, cadaverine, histamine, and spermidine reduction in sausage [40]; Lacticaseibacillus paracasei (formerly Lactobacillus paracasei) for histamine and tyramine reduction in cheese [41]; and Latilactobacillus curvatus (formerly Lactobacillus curvatus) for total BA reduction in fermented 
meat [42]. In addition, it was confirmed that the multicopper oxidase gene (MCO) found in LAB strains may be primarily responsible for BA degradation [38,41,42].

Although the capability of LAB strains to degrade BAs in fermented foods has been reported in previous studies regarding kimchi products, few reports are available on the BA degradation effects of BA-degrading LAB strains. Therefore, this study was performed to seek BA-degrading LAB that originated from kimchi and to pursue a mechanism of $B A$ degradation using a primer set that detects the $\mathrm{MCO}$ gene. The practical effects of the selected BA-degrading LAB strains on BA reduction during fermentation of Baechu kimchi were also investigated. This is the first study on the use of BA-degrading LAB strains originating from kimchi, which carry the $\mathrm{MCO}$ gene, as starter (or protective) cultures to reduce the content of BAs, including polyamines and toxic BAs, in kimchi.

\section{Materials and Methods}

\subsection{Bacterial Strains Used}

A total of $1448 \mathrm{LAB}$ strains were isolated from six types of kimchi varieties, including Baechu kimchi (napa cabbage kimchi), Kkakdugi (kimchi made mainly of diced radish), Chonggak kimchi (kimchi made mainly of ponytail radish), Yeolmu kimchi (kimchi made mainly of young radish), Gat kimchi (kimchi made mainly of mustard leaf), and $\mathrm{Pa}$ kimchi (kimchi made mainly of green onion), including those from previous studies (532 strains) [32,34] and in the present study (916 strains). The reference LAB strains used in this study, including Levilactobacillus brevis JCM 1170, L. helveticus KCCM 40989, L. plantarum KCTC 3108, L. sakei KCCM 43213, L. casei KCCM 12452, L. paracasei KCTC 3510, Limosilactobacillus fermentum KCTC 3112, Lentilactobacillus buchneri KCTC 5064, Leu. mesenteroides KCTC 3505, and Leu. citreum KCCM 12030, were purchased from the Korean Culture Center of Microorganisms (KCCM; Seoul, South Korea), the Japan Collection of Microorganisms (JCM; Saitama, Japan), and the Korean Collection for Type Cultures (KCTC; Daejeon, South Korea). All strains were cultured in de Man, Rogosa, and Sharpe (MRS; Laboratorios Conda, Madrid, Spain) broth at $37^{\circ} \mathrm{C}$ for $48 \mathrm{~h}$ and kept in glycerol $(20 \%, v / v)$ in a deep freezer at $-70{ }^{\circ} \mathrm{C}$.

\subsection{Determination of Histamine and Tyramine Degradation by LAB Strains}

Degradation of toxic BAs by LAB strains was assessed following the method developed by Leuschner et al. [43], with slight modifications. To prepare resting cells, $10 \mu \mathrm{L}$ of glycerol stock of each LAB strain were inoculated in $5 \mathrm{~mL}$ of MRS broth. After aerobic incubation at $37^{\circ} \mathrm{C}$ for $48 \mathrm{~h}$, a loopful of the cultured broth was streaked on MRS agar, which was incubated under the same conditions. A single colony was inoculated in $5 \mathrm{~mL}$ of MRS broth. After aerobic incubation at $37^{\circ} \mathrm{C}$ for $48 \mathrm{~h}, 200 \mu \mathrm{L}$ of the cultured broth was transferred into $10 \mathrm{~mL}$ of MRS broth. After aerobic incubation at $37^{\circ} \mathrm{C}$ for $48 \mathrm{~h}$ under shaking at $200 \mathrm{rpm}$, the cultured broth was centrifuged at $9000 \times g$ for $10 \mathrm{~min}$ at $4{ }^{\circ} \mathrm{C}$. The pellet was washed twice with sodium phosphate buffer $(0.05 \mathrm{M}, \mathrm{pH} 7.00$; $4.0962 \mathrm{~g}$ of $\mathrm{Na}_{2} \mathrm{HPO}_{4}$ and $2.537 \mathrm{~g}$ of $\mathrm{NaH}_{2} \mathrm{PO}_{4}$ dissolved in $1 \mathrm{~L}$ of distilled water, all from Sigma-Aldrich Chemical Co., St. Louis, MO, USA). The cell pellet was resuspended in $10 \mathrm{~mL}$ of the same buffer but that contained $0.5 \mathrm{mM}$ histamine and $0.5 \mathrm{mM}$ tyramine (all from Sigma-Aldrich, $w / v$ ). The cell suspension was reacted with shaking at $200 \mathrm{rpm}$ for $24 \mathrm{~h}$ at $30^{\circ} \mathrm{C}$, an optimal temperature for amine oxidase activity. The cell suspension was then filtered through a $0.2 \mu \mathrm{m}$ membrane filter (Millipore Co., Bedford, MA, USA) using a syringe and immediately analyzed by HPLC for toxic BA degradation.

To accelerate the screening of toxic BA-degrading LAB strains, an alternative method to measure toxic BA degradation by LAB strains in MRS broth was used [39]. Briefly, after activation of the LAB strains in MRS broth under the same incubation conditions above, $200 \mu \mathrm{L}$ of the cultured broth were inoculated into $10 \mathrm{~mL}$ of MRS broth supplemented with $50 \mathrm{ppm}(w / v)$ of histamine or tyramine. The MRS broth containing histamine or tyramine without bacterial cells was used as a control. All broths were incubated statically at $30^{\circ} \mathrm{C}$ for $24 \mathrm{~h}$. Subsequently, the broth culture was filtered through a $0.2 \mu \mathrm{m}$ membrane 
and one milliliter of the filtered broth culture was immediately analyzed using HPLC. After assessing the degradation activity of LAB strains using this method, selected LAB strains showing higher degradation activity were retested for degradation activity using the method of measurement in buffer as described above.

\subsection{Identification of $L A B$ Strains}

The LAB strains selected based on toxic BA degradation were identified through $16 \mathrm{~S}$ rRNA gene sequencing with universal bacterial primers $518 \mathrm{~F}$ and $805 \mathrm{R}$, as in the previous studies [32,34].

\subsection{Baechu Kimchi Fermentation with the Selected LAB Strains Capable of Degrading Toxic BAs}

To investigate the effects on the reduction in tyramine, histamine, and other BAs by selected LAB strains, Baechu kimchi was prepared using the recipe described by Kim et al. [44]. Napa cabbages were obtained from a grocery store in Sejong, South Korea. Napa cabbages were quartered and soaked in $10 \%$ salt brine $(w / v)$ for $10 \mathrm{~h}$. Then, salted napa cabbages were washed thrice with tap water. After draining for $3 \mathrm{~h}$ at room temperature, $100.0 \mathrm{~g}$ of salted napa cabbage were mixed with $13.0 \mathrm{~g}$ julienned white radish, $3.5 \mathrm{~g}$ red pepper powder, $2.2 \mathrm{~g}$ Myeolchi-aekjeot (Korean salted and fermented anchovy sauce), $2.0 \mathrm{~g}$ green onion, $1.4 \mathrm{~g}$ garlic, $1.0 \mathrm{~g}$ sugar, and $0.6 \mathrm{~g}$ ginger. The salinity of the kimchi was adjusted to $2.5 \%$ by adding deionized water.

The Baechu kimchi was divided into seven experimental groups-one non-inoculated group (C group) and six inoculated groups. The inoculated groups were LB, LT, LM, LP, and LS groups inoculated with each of the five selected LAB strains (L. brevis PK08, L. pentosus PK05, Leu. mesenteroides YM20, L. plantarum KD15, and L. sakei YM21, respectively) capable of degrading toxic BAs and incapable of producing both toxic BAs. The $\mathrm{C}$ group fermented naturally without inoculum and the PC group inoculated with L. brevis PK11, capable of largely producing tyramine $(295.63 \pm 12.44 \mu \mathrm{g} / \mathrm{mL})$, served as the control and positive control, respectively. Each LAB inoculum was inoculated into Baechu kimchi (except for the $\mathrm{C}$ group) at a level of about $7 \mathrm{Log} \mathrm{CFU} / \mathrm{g}$. All the experimental groups were placed in plastic containers $\left(250 \times 150 \times 200 \mathrm{~mm}^{3}\right)$ and fermented for three days at $25^{\circ} \mathrm{C}$, a temperature commonly used for kimchi fermentation experiments [34]. All kimchi samples belonging to the seven experimental groups were prepared in duplicate.

\subsection{Measurements of Physicochemical and Microbial Properties}

For all measurements of physicochemical and microbial properties, Baechu kimchi broth was sampled following previous studies [32,34,45]. The physicochemical properties, including $\mathrm{pH}$, acidity, salinity, and water activity, and the microbial properties, including the counts of total viable mesophilic bacteria and $\mathrm{LAB}$, of the samples from respective experiment groups were measured as in the previous studies $[32,34,45]$. Briefly, the $\mathrm{pH}$ and water activity $\left(\mathrm{a}_{\mathrm{w}}\right)$ of Baechu kimchi samples were determined using a $\mathrm{pH}$ meter (Orion 3-star Benchtop pH meter; Thermo Scientific, Waltham, MA, USA) and a water activity meter (AquaLab Pre; Meter Group, Inc., Pullman, WA, USA), respectively. The acidity and salinity of Baechu kimchi samples were determined according to the AOAC methods, respectively [46]. The counts of total viable mesophilic bacteria and LAB were determined on Plate Count Agar (PCA; Becton Dickinson, Sparks, MD, USA) and MRS agar, respectively.

\subsection{Analyses of BAs in Baechu Kimchi Samples and Assay Menstrua}

BA analyses in Baechu kimchi samples and assay menstrua were conducted according to protocols described in a previous study [34]. For an internal standard, 1,7Diaminoheptane ( $1 \mathrm{mg} / \mathrm{mL}$; Sigma) was used. Tyramine hydrochloride, histamine dihydrochloride, cadaverine dihydrochloride, putrescine dihydrochloride, spermine tetrahydrochloride, spermidine trihydrochloride, tryptamine, and $\beta$-phenylethylamine hydrochloride were used to prepare standard solutions (all from Sigma). The analysis procedures 
included extraction of BAs, preparation of BA standard solutions, derivatization of BAs, and chromatographic separation of BAs. Both limits of detection and limits of quantification for all BAs were about $0.10 \mu \mathrm{g} / \mathrm{mL}$ in standard solutions and $0.01-0.10 \mathrm{mg} / \mathrm{kg}$ and $0.02-0.31 \mathrm{mg} / \mathrm{kg}$, respectively, in food matrices [47].

\subsection{Detection and Identification of Multicopper Oxidase Gene in LAB Strains}

Polymerase chain reaction (PCR) was performed to detect a gene encoding a BAdegrading enzyme, $\mathrm{MCO}$, responsible for BA degradation in the LAB strains. To design specific gene primers, the DNA sequence of the MCO gene for L. brevis was retrieved from the National Center for Biotechnology Information (NCBI). The primer set, Lbre-F (5'-TGCCCGTTACGTGAGACTAC-3') and Lbre-R (5'-GACTTGTGCTGAACGTGCTG-3'), was designed for detection of the target gene in the L. brevis strains and in the other strains using the NCBI Primer-BLAST [48]. The PCR-amplified DNA fragment was 390 base pairs in length.

Afterwards, genomic DNA extraction from bacterial cultures was conducted using the G-spinTM genomic DNA extraction kit (Intron Biotechnology, Inc., Seongnam, South Korea). The $50-\mu \mathrm{L}$ PCR reaction mixture consisted of $1.5 \mu \mathrm{L}$ of $10 \mathrm{pM}$ of each primer, $0.25 \mu \mathrm{L}$ of Ex Taq DNA polymerase (5.0 units/ $\mu \mathrm{L}$; Takara Biotechnology Co. Ltd., Tokyo, Japan), $5 \mu \mathrm{L}$ of $10 \times$ Ex Taq buffer, $4 \mu \mathrm{L}$ of dNTPs (each at $0.2 \mathrm{mM}$; Takara), and $37.75 \mu \mathrm{L}$ of $200 \mathrm{ng}$ genomic DNA in deionized water. PCR amplification was carried out using a thermal cycler (DNA Engine Peltier T100 thermal cycler, Bio-Rad Laboratories, Hercules, CA, USA). The PCR conditions were as follows: an initial denaturation at $94{ }^{\circ} \mathrm{C}$ for $1 \mathrm{~min}$, followed by 30 cycles of denaturation at $94{ }^{\circ} \mathrm{C}$ for $30 \mathrm{~s}$, annealing at $54{ }^{\circ} \mathrm{C}$ for $40 \mathrm{~s}$ and extension at $72{ }^{\circ} \mathrm{C}$ for $1 \mathrm{~min}$, and final extension at $72{ }^{\circ} \mathrm{C}$ for $5 \mathrm{~min}$.

The PCR products were electrophoresed on agarose gel $(1.2 \%, w / v$; Duchefa Biochemie, Haarlem, The Netherlands) in 0.5× Tris-borate-EDTA buffer (TBE; Bio Basic, Markham, ON, Canada) with ethidium bromide $(0.5 \mu \mathrm{g} / \mathrm{mL}$; Sigma-Aldrich) by electrophoresis at $100 \mathrm{~V}$ for $30 \mathrm{~min}$. The $1 \mathrm{~kb}$ DNA Ladder (Elpis Biotech, Daejeon, South Korea) was used as a size marker. Subsequently, the gel was visualized on a transilluminator (SMU-01 Slider UV Imager; Maestrogen Inc., Hsinchu, Taiwan) under ultra-violet light and photographed.

The PCR products were also sequenced and compared with the MCO gene of L. brevis, available in the GenBank database using the Basic Local Alignment Search Tool [49].

\subsection{Statistical Analyses}

All physicochemical measurements were performed in triplicate, while microbial measurements were conducted in duplicate. Fermentation experiments with the seven experimental kimchi groups were conducted independently in duplicate. Data were presented as means and standard deviations of duplicates or triplicates. Error bars in all plots indicate the standard deviations determined from the fermentation experiments in duplicate. The determination of significant differences was conducted using the one-way analysis of variance (ANOVA) with Fisher's pairwise comparison in Minitab statistical software (Version 17.1.0. Minitab Inc., State College, PA, USA), and differences with a probability $(p)$ value of $<0.05$ were considered statistically significant.

\section{Results and Discussion}

\subsection{Histamine and Tyramine Degradation Activity of LAB Strains Isolated from Kimchi Products}

To screen LAB strains capable of degrading toxic BAs (i.e., histamine and tyramine), a total of $1448 \mathrm{LAB}$ strains, consisting of $532 \mathrm{LAB}$ strains used in previous studies [32,34] and kept in the laboratory, and 916 LAB strains newly obtained in the present study, isolated from six types of kimchi products, including Baechu kimchi, Kkakdugi, Chonggak kimchi, Yeolmu kimchi, Gat kimchi, and $\mathrm{Pa}$ kimchi, were tested for toxic BA degradation activity in a sodium phosphate buffer and/or in culture media containing BAs. 
Most of the LAB strains (1373 strains) tested displayed low or no degradation of toxic BAs, showing a degradation rate of less than $10 \%$ (Tables S1 and S2). Contrastingly, the other $75 \mathrm{LAB}$ strains showed the capability to degrade more than $10 \%$ of either toxic BA, and were subject to further testing, though only in culture media to observe the results quickly, to confirm the reproducibility of their degradation activity. As a result, five LAB strains exhibited reproducibility for a toxic BA degradation rate of $10 \%$ or more. Overall, the ranges of the highest degradation rates of toxic BAs by each of the five selected LAB strains were as follows: from 1.39 to $10.81 \%$ for histamine and from 7.43 to $14.97 \%$ for tyramine in buffer, and from 1.59 to $8.56 \%$ for histamine and from 11.68 to $14.88 \%$ for tyramine in culture media (Table 1). Through $16 \mathrm{~S}$ rRNA sequencing, the five selected LAB strains, PK08, PK05, YM20, KD15, and YM21, were identified as L. brevis (accession number NR116238), L. pentosus (accession number NR029133), Leu. mesenteroides (accession number NR118557), L. plantarum (accession number NR117813), and L. sakei (accession number NR042443), respectively (Table 1). The identified species have often been found in Baechu kimchi, and among them, L. brevis, Leu. mesenteroides, and L. plantarum have been especially considered to be representative LAB species associated with kimchi fermentation $[22,50,51]$. Considering the results described above, these five LAB strains were selected for further use in kimchi fermentation. In addition, all other strains identified as one of L. brevis, L. pentosus, Leu. mesenteroides, L. plantarum, and L. sakei, like the five selected LAB strains, showed lower degradation of both toxic BAs.

Table 1. Degradation rates of histamine and tyramine in buffer and media by selected LAB strains originated from kimchi varieties.

\begin{tabular}{|c|c|c|c|c|c|}
\hline \multirow{3}{*}{$\begin{array}{c}\text { Kimchi } \\
\text { Isolate No. }\end{array}$} & \multicolumn{2}{|c|}{ Buffer } & \multicolumn{2}{|c|}{ Media } & \multirow{3}{*}{ Identification } \\
\hline & \multicolumn{2}{|c|}{ Degradation (\%) } & \multicolumn{2}{|c|}{ Degradation (\%) } & \\
\hline & HIS $^{2}$ & TYR $^{2}$ & HIS & TYR & \\
\hline PK08 & $\begin{array}{c}3.65-8.58^{3} \\
(6.12 \pm 3.49)^{a}\end{array}$ & $\begin{array}{c}3.50-11.54 \\
(7.52 \pm 5.69)^{\mathrm{a}}\end{array}$ & $\begin{array}{c}5.26-8.56 \\
(6.91 \pm 2.33)^{a}\end{array}$ & $\begin{array}{c}1.82-11.68 \\
(6.75 \pm 6.97)^{\mathrm{a}}\end{array}$ & Levilactobacillus brevis \\
\hline PK05 & $\begin{array}{c}7.47-10.81 \\
(9.14 \pm 2.36)^{\mathrm{a}}\end{array}$ & $\begin{array}{c}5.25-7.43 \\
(6.34 \pm 1.54)^{\mathrm{a}}\end{array}$ & $\begin{array}{c}1.17-1.59 \\
(1.38 \pm 0.30)^{b}\end{array}$ & $\begin{array}{c}2.97-13.13 \\
(8.05 \pm 7.18)^{\mathrm{a}}\end{array}$ & Lactiplantibacillus pentosus \\
\hline YM20 & $\begin{array}{c}\text { ND-1.39 } \\
(0.70 \pm 0.98)^{b}\end{array}$ & $\begin{array}{c}3.25-11.90 \\
(7.58 \pm 6.12)^{a}\end{array}$ & $\begin{array}{c}4.09-4.28 \\
(4.19 \pm 0.13)^{\mathrm{a}}\end{array}$ & $\begin{array}{c}2.11-14.36 \\
(8.24 \pm 8.66)^{\mathrm{a}}\end{array}$ & Leuconostoc mesenteroides \\
\hline YM21 & $\begin{array}{c}0.21-10.54 \\
(5.38 \pm 7.30)^{a}\end{array}$ & $\begin{array}{c}3.47-11.41 \\
(7.44 \pm 5.61)^{\mathrm{a}}\end{array}$ & $\begin{array}{c}0.58-7.96 \\
(4.27 \pm 5.22)^{\mathrm{a}}\end{array}$ & $\begin{array}{c}2.39-12.11 \\
(7.25 \pm 6.87)^{\mathrm{a}}\end{array}$ & Latilactobacillus sakei \\
\hline KD15 & $\begin{array}{c}3.64-5.67 \\
(4.66 \pm 1.44)^{\mathrm{a}}\end{array}$ & $\begin{array}{c}4.03-14.97 \\
(9.50 \pm 7.74)^{\mathrm{a}}\end{array}$ & $\begin{array}{c}\text { ND-4.34 } \\
(2.17 \pm 3.07)^{\mathrm{a}}\end{array}$ & $\begin{array}{c}2.39-14.88 \\
(8.64 \pm 8.83)^{\mathrm{a}}\end{array}$ & Lactiplantibacillus plantarum \\
\hline
\end{tabular}

${ }^{1} \mathrm{PK}: \mathrm{Pa}$ kimchi (kimchi made mainly of green onion), YM: Yeolmu kimchi (kimchi made mainly of young radish), KD: Kkakdugi (kimchi made mainly of diced radish). ${ }^{2}$ HIS: histamine, TYR: tyramine. ${ }^{3}$ Values represent the minimum and maximum (mean \pm standard deviation) as determined by duplicate experiments. Mean values in the same row of the same BA followed by a different letter (a-b) are significantly different $(p<0.05)$, ND: no degradation detected.

Regarding the assay menstrua, while determination in buffer has been shown to precisely measure degradation rates, determination in culture media can obtain results more quickly $[39,43]$. Although the former may be considered more reliable, there was no statistically significant difference between the results of almost all the degradation rates obtained from both the buffer and culture media (Table 1). Based on these results, it seems preferable to use the latter to quickly select toxic BA-degrading LAB strains.

Alvarez and Moreno-Arribas [52] reviewed that foodborne bacteria, such as Lactobacillus spp., Pediococcus spp., Rhodococcus spp., Arthrobacter spp., Micrococcus spp., Brevibacterium spp., Bacillus spp., Staphylococcus spp., and Oenococcus spp., could degrade BAs using their amine-oxidation activity. Dapkevicius et al. [39] found that 4 of 48 LAB strains isolated from fish pastes were capable of degrading histamine in assay broth, showing a degradation rate ranging from 19.61 to $56.18 \%$, and were all identified as L. sakei. Herrero-Fresno et al. [53] reported that 17 of $157 \mathrm{LAB}$ strains isolated from cheeses degraded histamine 
and tyramine in the ranges of 12.75 to $47.90 \%$ and 14.92 to $56.52 \%$, respectively, in broth culture, and were all identified as Lacticaseibacillus casei (formerly Lactobacillus casei). The previous studies are in agreement with this study, showing the capability of LAB strains isolated from kimchi products to degrade toxic BAs. In this study, however, the capability of degrading tyramine or histamine by the selected LAB strains appeared to be slightly lower than that of LAB strains reported in the aforementioned studies. The BA content of kimchi may be one of the reasons for the difference in the degradation activity of LAB strains in the current study compared to previous studies. It is known that the BA content in kimchi is low compared to other fermented foods, likely due to the fact that the amount of precursor amino acids of BAs is lower in kimchi than in protein-rich fermented foods such as cheese, fermented seafood products, fermented soybean foods, fermented sausage, etc., which provide abundant precursor amino acids [29-31,37]. Therefore, it seems inevitable that the BA degradation activity of LAB strains isolated from kimchi with small amounts of degradable BAs would be relatively low. To isolate LAB strains possessing a strong capability to degrade BAs from kimchi, steady efforts and research are likely required in the future. In addition, to prove that the difference in the BA degradation activity of LAB is at least partly due to the degradable BA content of the foods (i.e., protein-poor and protein-rich foods) from which they are isolated, a survey study comparing the BA degradation activity of LAB isolated from various foods with different protein content is also required.

Meanwhile, the BA-producing ability of the five LAB strains selected in this study had previously been examined by Jin et al. [32] and Lee et al. [34]. In the previous reports, the five LAB strains displayed low (below $3 \mu \mathrm{g} / \mathrm{mL}$ ) or no production of eight kinds of BAs, and, particularly, no production of both toxic BAs. Although one of the five LAB strains was identified as L. brevis, the other LAB strains not included in the five strains but identified as L. brevis were found to produce large quantities of BAs. Together with the toxic BA degradation results of the L. brevis PK08 strain described above, the results of these studies support other previous reports suggesting that BA-producing and toxic BA-degrading capabilities depend not only on the species but also on the strains. Consequently, the five LAB strains capable of degrading toxic BAs and incapable of producing both toxic BAs were isolated from kimchi varieties in this study. It is expected that BA concentrations in kimchi could be reduced by the use of the selected LAB strains as starter or protective cultures in fermentation.

3.2. Changes in Physicochemical and Microbiological Parameters during Fermentation of Baechu Kimchi Inoculated with BA-Degrading LAB Strains

Fermentation of Baechu kimchi was carried out to practically investigate the influences of the strains selected above (L. brevis PK08, L. pentosus PK05, Leu. mesenteroides YM20, L. plantarum KD15, and L. sakei YM21) on the physicochemical and microbial properties (Section 3.2) as well as on the BA content in kimchi (Section 3.3).

The changes in physicochemical properties (Figure 1) of the non-inoculated group (C group, naturally fermented control) and all inoculated groups (PC group, positive control inoculated with a tyramine-producing LAB strain; LB, LT, LM, LP, and LS groups inoculated with toxic BA-degrading LAB strains; refer to Section 2.4) were consistent with those of a previous study described by Mheen and Kwon [54], which reported on kimchi fermented under conditions similar to the current study. 
(a)

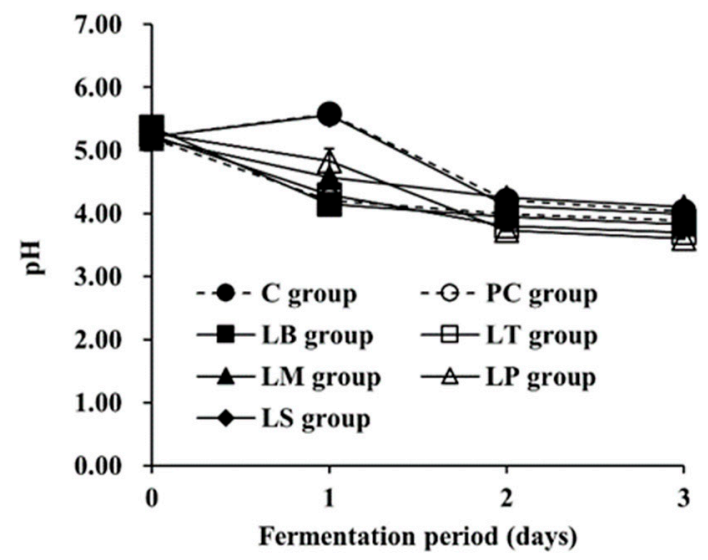

(b)

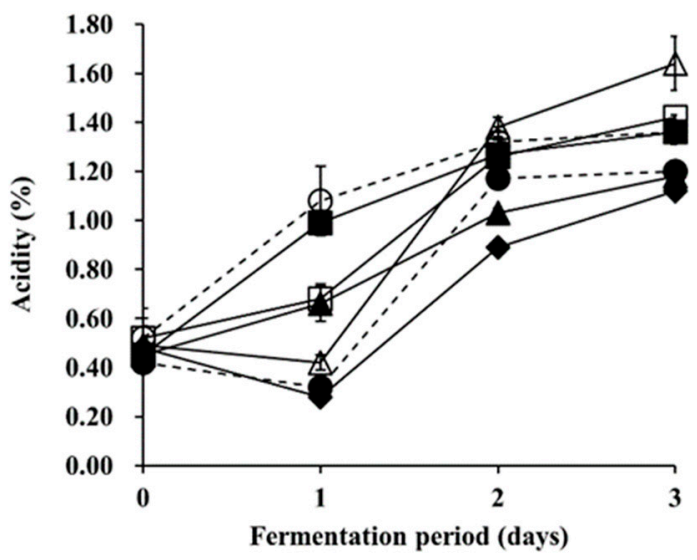

Figure 1. Changes in physicochemical properties of Baechu kimchi during fermentation. (a) $\mathrm{pH}$, (b) acidity. •: C group without an inoculum (control), $\bigcirc$ : PC group inoculated with tyramine-producing L. brevis isolated from kimchi product (positive control), $\mathbf{\square}$ : LB group inoculated with BA-degrading L. brevis PK08, $\square$ : LT group inoculated with BA-degrading L. pentosus PK05, $\mathbf{\Delta}$ : LM group inoculated with BA-degrading Leu. mesenteroides YM20, $\triangle$ : LP group inoculated with BA-degrading L. plantarum KD15, $\bullet$ : LS group inoculated with BA-degrading L. sakei YM21.

The initial $\mathrm{pH}$ values of all groups were determined to be in the range of $5.19 \pm 0.04$ to $5.37 \pm 0.16$ (5.24 \pm 0.06 , mean \pm standard deviation from the values of all the groups), as shown in Figure 1a. The $\mathrm{pH}$ of the $\mathrm{C}$ and LS groups increased slightly during the first day of fermentation, and thereafter decreased steadily to $4.03 \pm 0.04$ and $3.99 \pm 0.02$, respectively. This slight increase in $\mathrm{pH}$ is consistent with previous reports describing the fermentation of other kimchi varieties [32,34]. Meanwhile, the $\mathrm{pH}$ of the other groups (PC, LB, LT, LM, and LP groups) decreased gradually to the range of $3.60 \pm 0.01$ to $4.10 \pm 0.04$ throughout the fermentation period. The differences in $\mathrm{pH}$ variation among the groups as fermentation progressed is likely connected with the counts of total viable mesophilic bacteria, particularly LAB, as seen in Figure 2. In this regard, it is notable that the rise in microbial counts of the $C$ and LS groups on day one was slightly slower than those of the other groups (PC, LB, LT, LM, and LP groups). Thus, it seems that slower microbial growth, compared to the other groups, caused delayed production of organic acids, which in turn, affected the $\mathrm{pH}$ (and acidity, as well) of both groups.

Meanwhile, as shown in Figure $1 b$, the initial titratable acidity of all groups was in the range of $0.42 \pm 0.04$ to $0.52 \pm 0.12 \%(0.48 \pm 0.04 \%$, mean \pm standard deviation from the values of all the groups). As for the C and LS groups, the titratable acidity was observed to decrease slightly on the first day of fermentation and then increased gradually to $1.20 \pm 0.02 \%$ and $1.12 \pm 0.02 \%$, respectively, towards the end of the fermentation process. Contrastingly, the titratable acidity of the LP group remained statistically unchanged (but decreased slightly) on day one compared to the initial acidity and rose thereafter, while that of the PC, LB, LT, and LM groups increased throughout the whole fermentation period. On day three of fermentation, the titratable acidity of the PC, LB, LT, LM, and LP groups eventually reached a range of $1.18 \pm 0.06$ to $1.64 \pm 0.11 \%$. These changes in acidity throughout the fermentation period corresponded with the changes in $\mathrm{pH}$ for all groups. In other words, a rise in $\mathrm{pH}$ was seen concurrently with a decrease in acidity, and vice versa. It is also worth noting that the largest increases in acidity on day one were seen in the PC and LB groups, while the LP group displayed the largest acidity increase each day thereafter. In any case, the titratable acidity of all groups reached over $1.0 \%$ by the third day of fermentation, indicating that the Baechu kimchi was over-ripened. Kimchi ripeness is commonly divided into less ripened, optimally ripened, and over-ripened, based on $\mathrm{pH}$ and acidity. Mheen and Kwon [54] suggested that the $\mathrm{pH}$ and acidity of optimally ripened kimchi are in the ranges of 4.2 to 4.5 and 0.6 to $1.0 \%$, respectively, while the acidity of overripened kimchi is over $1.0 \%$. According to these criteria, the PC group had already reached 
the over-ripened level on day one. Meanwhile, optimal fermentation of the LB, LT, and LM groups was observed on the first day, and on day two for the LS group. Interestingly, the LP group was less ripened on day one, but found to be over-ripened on day two, without observation of a state of optimal ripening. The $C$ group followed a similar pattern as the LP group. Thus, it is expected that kimchi fermentation can be controlled by the use of selected LAB strains with specific effects on kimchi acidity, to give a final product with a desired level of ripeness for any given length of fermentation.
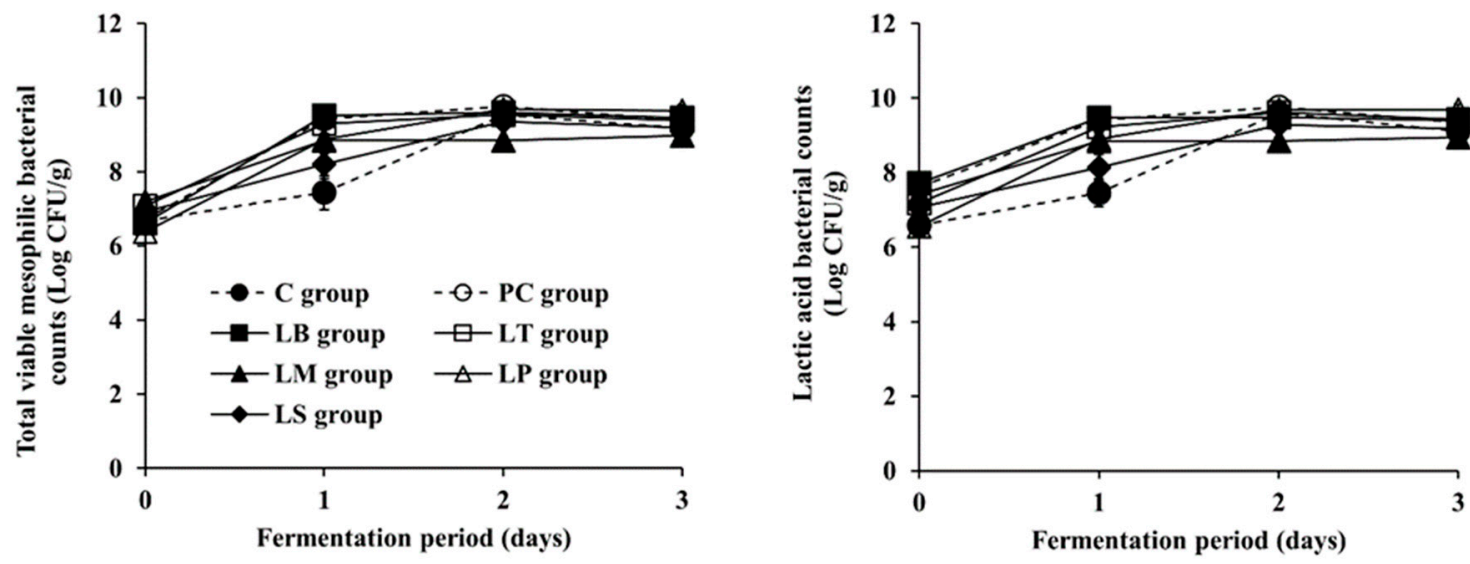

Figure 2. Changes in microbial properties of Baechu kimchi during fermentation. •: C group without an inoculum (control), $\bigcirc$ : PC group inoculated with tyramine-producing L. brevis isolated from kimchi product (positive control), $\mathbf{\square}$ : LB group inoculated with BA-degrading L. brevis PK08, $\square$ : LT group inoculated with BA-degrading L. pentosus PK05, $\mathbf{\Delta}$ : LM group inoculated with BA-degrading Leu. mesenteroides YM20, $\triangle$ : LP group inoculated with BA-degrading L. plantarum KD15, $\bullet$ : LS group inoculated with BA-degrading L. sakei YM21.

The inoculum size for all inoculated groups was at the same level of $7 \mathrm{Log} C F U / \mathrm{mL}$, but the initial counts of total viable mesophilic bacteria and LAB immediately after Baechu kimchi preparation were somewhat different among the groups, as shown in Figure 2. In detail, the initial total viable mesophilic bacteria and LAB counts of the $C$ group were $6.67 \pm 0.06 \mathrm{Log} C F U / \mathrm{mL}$ and $6.59 \pm 0.13 \mathrm{Log} C F U / \mathrm{mL}$, respectively. The LP group showed the lowest initial counts of total viable mesophilic bacteria and LAB, $6.38 \pm 0.06 \mathrm{Log}$ $\mathrm{CFU} / \mathrm{mL}$ and $6.56 \pm 0.09 \mathrm{Log} \mathrm{CFU} / \mathrm{mL}$, while those of the other inoculated groups (PC, LB, LT, LM, and LS groups) were higher than the C group by up to $0.53 \mathrm{Log} \mathrm{CFU} / \mathrm{mL}$ and $1.13 \mathrm{Log} C F U / \mathrm{mL}$, respectively. Therefore, considering that the inoculum size for each group was at the same level, the adaptability of LAB strains to the kimchi environment seems to differ depending on the species and/or strains, which may eventually lead to different initial counts of total viable mesophilic bacteria and LAB. In any case, the counts of all groups increased throughout the kimchi fermentation. However, on the first day of fermentation, the counts of the C and LS groups were $7.44 \pm 0.46$ and $8.20 \pm 0.38 \mathrm{Log} \mathrm{CFU} / \mathrm{mL}$ for total viable mesophilic bacteria, respectively, and $7.46 \pm 0.37$ and $8.13 \pm 0.35 \mathrm{Log} C F U / \mathrm{mL}$ for LAB, respectively, while those of the other groups (PC, LB, LT, LM, and LP groups) were higher, in the ranges of $8.85 \pm 0.06$ to $9.51 \pm 0.10 \mathrm{Log}$ $\mathrm{CFU} / \mathrm{mL}$ for total viable mesophilic bacteria and $8.84 \pm 0.03$ to $9.47 \pm 0.09 \mathrm{Log} \mathrm{CFU} / \mathrm{mL}$ for LAB. Nevertheless, the counts of total viable mesophilic bacteria and LAB of the C and LS groups increased faster than the other groups between the first and second days of fermentation. Thus, the counts of total viable mesophilic bacteria and LAB of all groups reached the ranges of $8.85 \pm 0.07$ to $9.76 \pm 0.07 \mathrm{Log} \mathrm{CFU} / \mathrm{mL}$ and $8.84 \pm 0.05$ to $9.76 \pm 0.06 \mathrm{Log}$ $\mathrm{CFU} / \mathrm{mL}$, respectively, by day two of fermentation, and remained constant thereafter. These results support the assumption that slower microbial growth observed in both the C and LS groups might result in the highest $\mathrm{pH}$ and the lowest acidity, as aforementioned. In addition, in all groups, the counts of the total viable mesophilic bacteria were observed to be similar to those of the LAB during the fermentation period, which indicates that the 
LAB were the predominant microorganisms in the Baechu kimchi. Altogether, the results of physicochemical and microbial measurements indicate that all Baechu kimchi groups, regardless of inoculum, were properly fermented.

\subsection{Changes in BA Content during Fermentation of Baechu Kimchi Inoculated with $B A$-Degrading LAB Strains}

Through the Baechu kimchi fermentation experiments described in Section 3.2, it was also evaluated whether the selected LAB strains with the capability of toxic BA degradation would practically reduce the content of BAs (particularly tyramine) in Baechu kimchi. Changes in the content of six kinds of BAs, including tyramine, histamine, putrescine, cadaverine, spermidine, and spermine, during Baechu kimchi fermentation, are presented in Figures 3, 4 and S1 and Table S3. Tryptamine and $\beta$-phenylethylamine were also determined but are not described hereafter, as the tryptamine content in all groups was lower than $10 \mathrm{mg} / \mathrm{kg}$ throughout the fermentation period, and $\beta$-phenylethylamine was not detected in all groups during fermentation (data not shown).
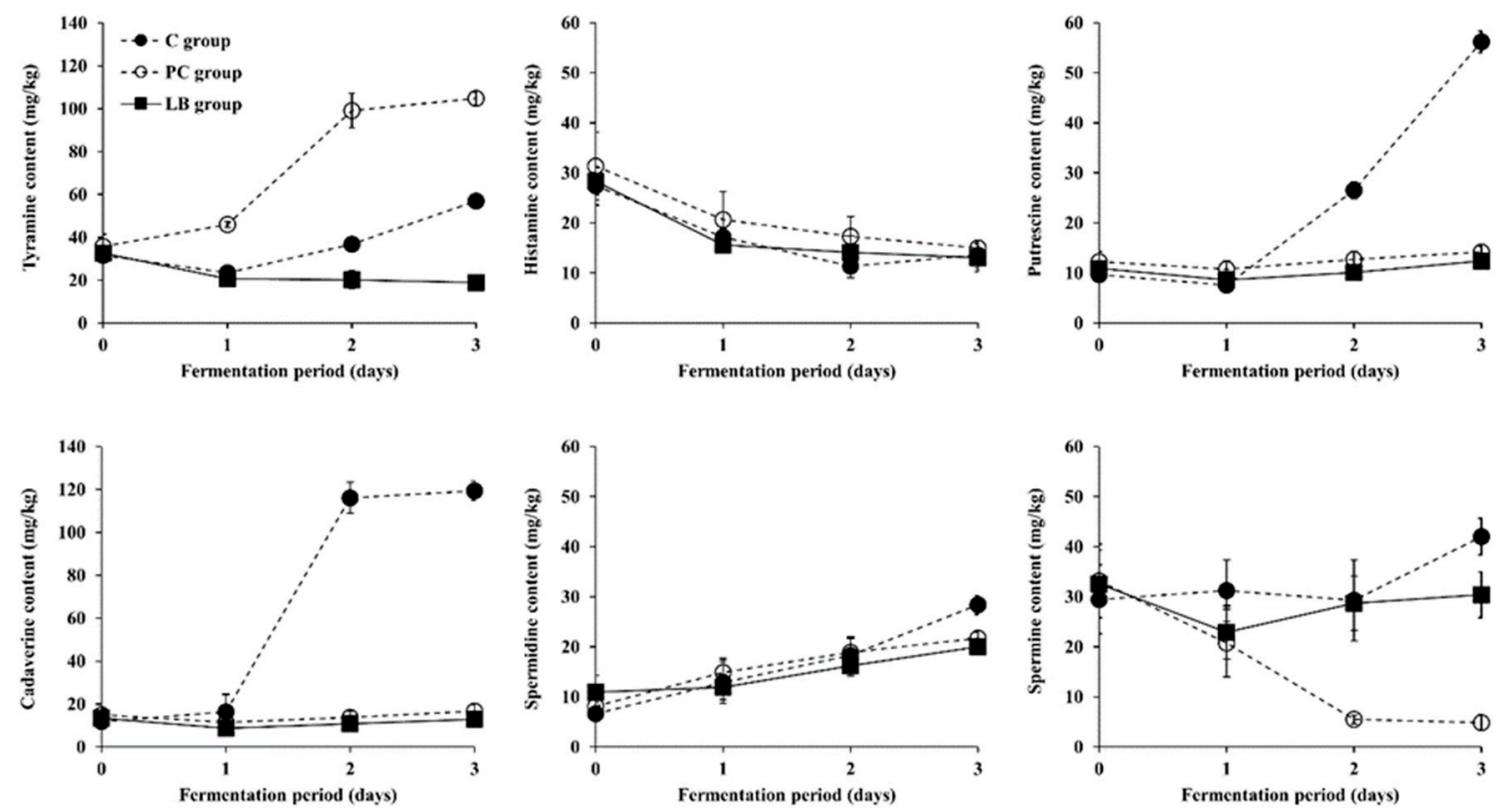

Figure 3. Changes in BA content in Baechu kimchi inoculated with a LAB strain leading to significant tyramine reduction during fermentation. $\bullet$ : C group without an inoculum (control), $\bigcirc$ : PC group inoculated with tyramine-producing L. brevis isolated from kimchi product (positive control), $\mathbf{0}$ : LB group inoculated with BA-degrading L. brevis PK08.

As shown in Figure 3, the tyramine, putrescine, cadaverine, spermidine, and spermine content of the $\mathrm{C}$ group (naturally fermented control without any inoculum) consistently increased between days one and three of fermentation, while the histamine content steadily deceased throughout the fermentation period. On day three of fermentation, this increased content of the five BAs of the $\mathrm{C}$ group was the highest among all the experimental Baechu kimchi groups (except for tyramine content in the PC and LS groups described below; also refer to Figures 3 and S1, respectively), but the decrease in histamine content was similar to the inoculated groups. These results were likely associated with the BA-producing and BA-degrading activities of some indigenous LAB strains, and are in accordance with observations from previous reports [32,34]. As suggested by Dierick et al. [55] and reviewed by Park et al. [30] and Barbieri et al. [56], the accumulation of BAs in fermented food products results from the decarboxylation of free amino acids by the amino acid-decarboxylase activity of some LAB species present in the food. Furthermore, other previous studies have reported that LAB species, including L. plantarum, L. brevis, L. curvatus, Lentilactobacillus hilgardii (formerly Lactobacilus hilgardii), L. casei, and Leu. mesenteroides, were likely responsi- 
ble for BA production in fermented foods such as wine, cheese, cider, beer, and kimchi, and had genes encoding amino acid decarboxylases [50,57-60]. Therefore, BA accumulation seems to be an unavoidable phenomenon in naturally fermented kimchi.

Meanwhile, the tyramine, putrescine, cadaverine, and spermidine content of the PC group inoculated with the tyramine-producing L. brevis PK11 strain steadily increased during the fermentation period, while histamine and spermine content gradually decreased (Figure 3). Among the four BAs that increased, the tyramine content of the PC group $(104.74 \pm 3.04 \mathrm{mg} / \mathrm{kg})$ on day three of fermentation was at least two times higher than that of the other groups (for instance, $56.88 \pm 1.25 \mathrm{mg} / \mathrm{kg}$ for C group) and was over the harmful level $(100 \mathrm{mg} / \mathrm{kg})$ of tyramine [8]. The observation was most likely due to the L. brevis PK11 strain being used as a tyramine-producing reference strain (but incapable of producing the other BAs). The content of putrescine, cadaverine, and spermidine was lower in the PC group than in the C group on day three. L. brevis PK11 is hence believed to be implicated in this difference, as the polyamine-producing strains present in the naturally fermented Baechu kimchi (C group) might have been restricted by this inoculated strain in the PC group, and thus not be able to produce the aforementioned BAs. In the meantime, the decrease in histamine content of the PC group throughout the fermentation period was consistent with that of the $C$ group. The spermine content of the PC group on days two and three, however, was the lowest among the groups. On the aspect of correlation between the changes in tyramine and spermine content of the PC group, interestingly, as the spermine content decreased during fermentation, the tyramine content increased $\left(R^{2}=0.93\right)$. A similar correlation has also been observed in previous studies [32,34]. Thus, the microbial production of tyramine seems to be negatively related to spermine content in Baechu kimchi. Further research is required to elucidate this assumption. In regard to the BA content of kimchi, only a small number of studies have been conducted [30]. Nevertheless, some reports have described that BA content in kimchi is within the acceptable ranges for human health $[29,61]$, however, kimchi with BA levels over the acceptable ranges has also been reported $[31,33,35]$. In addition, the current commercial production of kimchi depends largely on natural fermentation, because the application of starter cultures may affect the flavor and quality of kimchi products and there is no scientific guideline for the kimchi manufacturing process, such as the salting of napa cabbage and the ripening of the kimchi product $[62,63]$. Besides, considerable tyramine content was measured in the PC group inoculated with tyramine-producing L. brevis PK11, as mentioned above. Consequently, the presence of $\mathrm{LAB}$ strains possessing the ability to produce BAs, such as L. brevis PK11, isolated from a kimchi variety in this study, in kimchi products may lead to the accumulation of BAs as kimchi fermentation progresses, which may potentiate food safety risks associated with BAs (particularly tyramine). For this reason, efforts to find or develop BA-degrading LAB strains with weak or no BA-producing capability to be used as starter or protective cultures for kimchi fermentation are suggested for further research to reduce the content of BAs in kimchi products.

In contrast to the $\mathrm{C}$ and $\mathrm{PC}$ groups, as shown in Figure 3, the content of both tyramine and histamine of the LB group gradually decreased during fermentation. The cadaverine and spermine content also slightly, but statistically insignificantly, decreased, while the putrescine and spermidine content slightly, but statistically significantly, increased throughout the fermentation period. Overall, the content of the six BAs at the end of the fermentation period was remarkably lower in the LB group than in both the $C$ group and the PC group (except for spermine content in the PC group). Particularly, the tyramine content was reduced by $66.65 \%$ and $81.89 \%$ compared to the $C$ and PC groups, respectively. As discussed in Section 3.1, the five selected LAB strains had the potential to be used as toxic BA-degrading starter or protective cultures for the reduction in BA content in kimchi fermentation. Indeed, the toxic BA degradation capability of the L. brevis PK08 strain was validated not only in the in vitro BA degradation test (Section 3.1) but also in the Baechu kimchi fermentation experiment (Section 3.3). As for the change in tyramine content, a considerable decrease was observed in the LB group compared to that of the 
$\mathrm{C}$ and PC groups. Possible explanations for this result may include: (i) antimicrobial activity against BA-producing microorganisms, (ii) inactive BA production, and/or (iii) BA degradation activity of $L$. brevis PK08 strain. Regarding the first assumption, although the antimicrobial activity of all the selected LAB strains, including L. brevis PK08 strain, was tested in this study, none of the LAB strains were found to have antimicrobial activity against BA-producing LAB strains (data not shown). Thus, it seems that the other reasons are more probable. As for the change in histamine content, however, a gradual decrease during fermentation was observed in all groups, including the $C$ group that was fermented naturally. Also, as presented in Table 1, the histamine degradation of the L. brevis PK08 strain (and the other selected LAB strains) was lower than the tyramine degradation in both buffer and culture media. Therefore, it is unclear whether the decrease in histamine content of the LB group is attributed to degradation activity of the inoculated strain, L. brevis PK08. This is also the case for the other inoculated groups. Although further research on the kinetics and mechanism of histamine degradation during kimchi fermentation is suggested, it is evident that the L. brevis PK08 strain isolated from a kimchi variety somehow showed effective toxic BA degradation activity (particularly tyramine) during Baechu kimchi fermentation. It is also notable that the accumulation of all six BAs, including polyamines as well as toxic BAs, was suppressed in the LB group, compared to the $C$ group that was fermented naturally, as described above. Similar to the PC group, it seems that the polyamine-producing strains present in the naturally fermented Baechu kimchi were somehow restricted by the L. brevis PK08 strain in the LB group. Altogether, the present study suggests that LAB strains capable of degrading toxic BAs, such as L. brevis PK08, may be worthwhile to use for the prevention of food safety issues associated not only with toxic BAs but also with polyamines in kimchi.
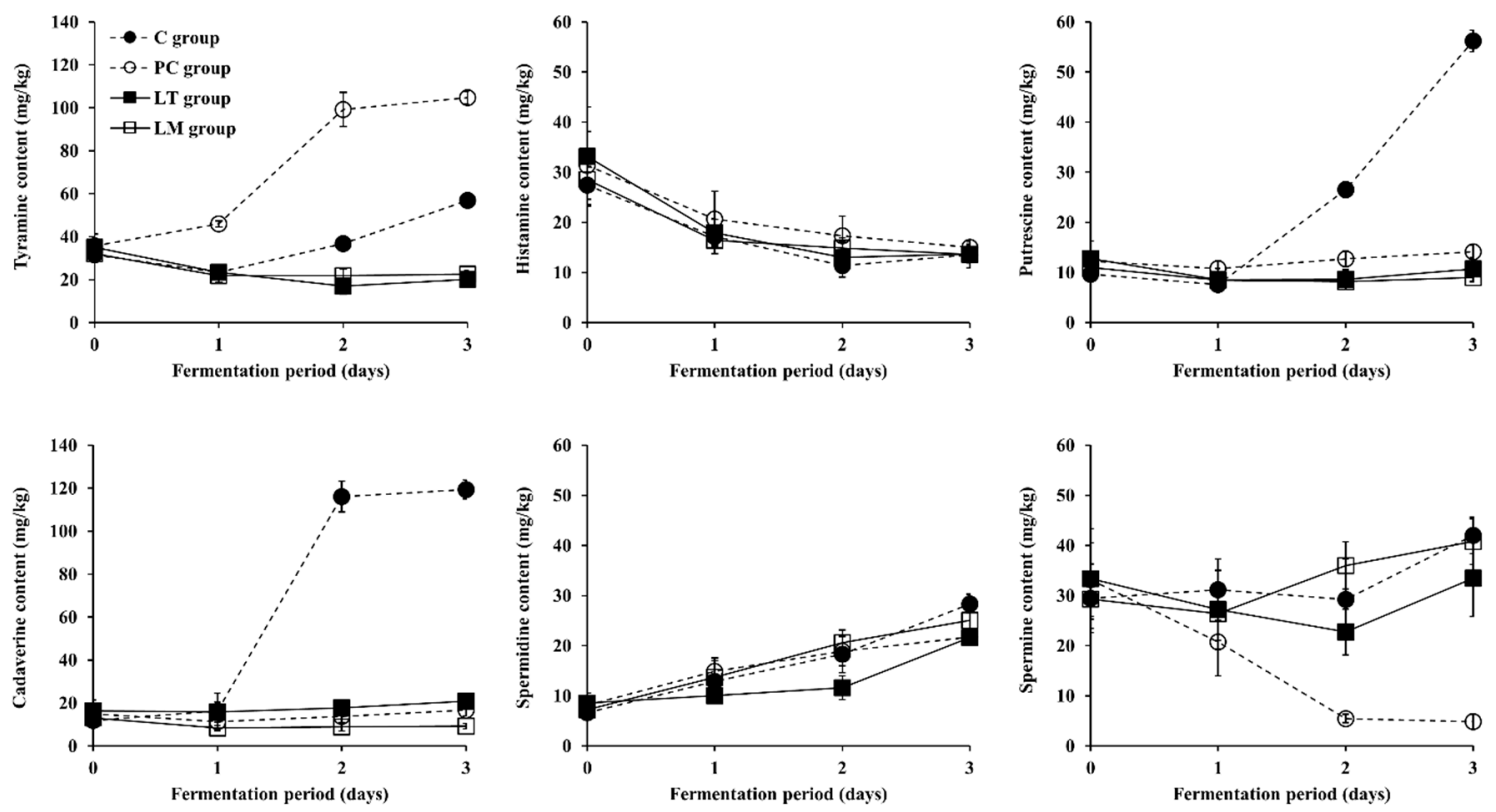

Figure 4. Changes in BA content in Baechu kimchi inoculated with LAB strains leading to moderate tyramine reduction during fermentation. $\bullet$ : C group without an inoculum (control), $\bigcirc$ : PC group inoculated with tyramine-producing $L$. brevis isolated from kimchi product (positive control), $\mathbf{\square}$ : LT group inoculated with BA-degrading L. pentosus PK05, $\square$ : LM group inoculated with BA-degrading Leu. mesenteroides YM20.

As shown in Figure 4, the changes in BA content of the LT and LM groups, inoculated with the L. pentosus PK05 strain or the Leu. mesenteroides YM20 strain, respectively, were determined to be similar to the patterns observed in the LB group. However, at the end of fermentation, the tyramine, histamine, spermidine, and spermine content of both the LT and LM groups were slightly higher than those of the LB group, while the putrescine 
content was slightly lower. Compared to the LB group, the cadaverine content was slightly higher in the LT group but slightly lower in the LM group. Nevertheless, the differences in each of the six BA contents were statistically insignificant among the three groups. Meanwhile, the LP and LS groups inoculated with the L. plantarum KD15 strain or the $L$. sakei YM21 strain, respectively, were also tested, but are not described hereafter because the groups did not show a strong effect on BA degradation activity in Baechu kimchi compared to the other groups (LB, LT, and LM groups; Figure S1). Based on the in vitro toxic BA degradation test (Table 1), it was expected that $L$. sakei YM21 would have moderate degradation activity of both tyramine and histamine in the Baechu kimchi fermentation experiment, and L. plantarum KD15 and Leu. mesenteroides YM20, which showed the highest and second-highest tyramine degradation activity in vitro, would also perform highly in fermentation experiments. Contrary to this expectation, L. brevis PK08 showed the most remarkable toxic BA degradation (particularly tyramine), while L. sakei YM21, L. plantarum KD15, and Leu. mesenteroides YM20 underperformed. Such results indicate that the use of only in vitro testing has limitations in selecting the optimal LAB strains capable of degrading BAs in practical fermentation. Therefore, as in this study, the best strategy for choosing an optimal strain for fermentation would be to shortlist a few high performing strains from the in vitro tests and apply them to the fermentation experiments, then select the optimal strain. In addition, since the level of Baechu kimchi ripeness at a given time differs due to how inocula of different LAB species affect acidity (as described in Section 3.2), it can be expected that the control of kimchi fermentation by the use of selected $\mathrm{LAB}$ strains as starter and/or protective cultures can not only reduce the BA content of the resulting product, but a favorable level of ripeness at a desired time can also be achieved.

\subsection{Contribution of Multicopper Oxidase Gene in L. brevis Strains to BA Degradation}

Previous studies have reported that LAB strains such as L. curvatus, L. plantarum, and L. paracasei could degrade BAs, including tyramine, histamine, and putrescine, in fermented foods such as fermented meat, wine, and cheese [38,41,42]. These studies have also described that the multicopper oxidase (MCO) genes which encode enzymes responsible for BA degradation are present in the LAB strains. The activity of such BAdegrading enzymes including MCOs and amine oxidases may provide substances that can be used as sources of energy and growth [64]. Other amine oxidase genes in LAB, such as monoamine oxidase, polyamine oxidase, and copper-containing amine oxidases, have rarely been registered in NCBI. As shown in Figure 5, therefore, it was examined whether the strain with the highest BA-degrading activity from the Baechu kimchi fermentation experiment, L. brevis PK08 (and L. brevis PK11 and L. brevis JCM 1170), carries the gene coding for a BA-degrading MCO. Using the primer set described in Section 2.7, the MCO gene was detected in all three L. brevis strains, but not detected in the other nine LAB species examined. The sequences of the detected genes showed high levels of identity (higher than 99\%) with L. brevis MCO gene (accession number CP031208.1). Thus, it is clear that MCO is responsible for BA degradation (particularly tyramine) of the L. brevis strains during Baechu kimchi fermentation in this study. It is noteworthy that glyceraldehyde-3-phosphate dehydrogenase (GAPDH) has been identified in L. plantarum as an enzyme capable of degrading histamine [65]. Together with the fact that GAPDH is produced by L. brevis (accession number QCZ55221), both MCO and GAPDH may have a role in BA degradation in kimchi. However, there is still a lack of available information on amine oxidase genes in LAB species, with the exception of MCO and GAPDH, as aforementioned. Apart from this, since the MCO genes are found in the genomes of L. pentosus, Leu. mesenteroides, L. plantarum, and L. sakei that are registered in NCBI, it is likely that in a future study, a universal-primer or multi-primer set could be developed for the rapid and simultaneous detection of the MCO genes in the LAB species. 


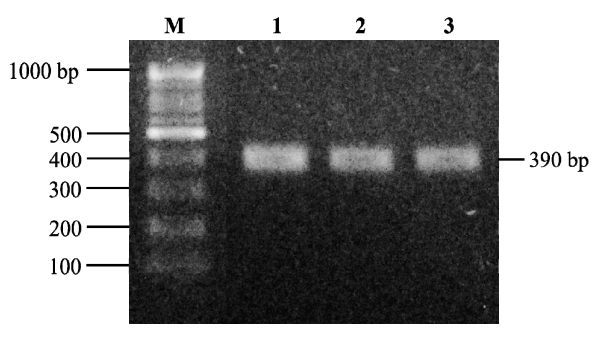

Figure 5. Electrophoresis of PCR products amplified for a gene encoding MCO (390 bp) in L. brevis. Lanes M: 1000 bp DNA ladder, 1: L. brevis JCM 1170, 2: L. brevis PK08, 3: L. brevis PK11. The MCO gene was not detected in L. helveticus KCCM 40989, L. plantarum KCTC 3108, L. sakei KCCM 43213, L. casei KCCM 12452, L. paracasei KCTC 3510, L. fermentum KCTC 3112, L. buchneri KCTC 5064, Leu. mesenteroides KCTC 3505, and Leu. citreum KCCM 12030.

Although L. brevis PK11 carried the MCO gene and showed tyramine-degrading activity in the toxic BA degradation test (data not shown), used as the positive control (PC group) in the Baechu kimchi fermentation experiment, tyramine content increased considerably. This indicates that when selecting a starter or protective culture that reduces the BA content of a fermented food product, in addition to BA degrading activity, BA production must also be considered.

\section{Conclusions}

In the present study, the highest BA levels (except for tyramine) among all the experimental Baechu kimchi groups were seen in the $C$ group, fermented naturally without a LAB inoculum, which seems to be associated with the BA-producing activity of those indigenous $\mathrm{LAB}$ species and/or strains present in the kimchi that are commonly responsible for BA production in lactic acid fermented foods, as reported in other previous studies. Based on observations in previous and present studies, BA accumulation seems to be inevitable in naturally fermented kimchi. In addition, the tyramine content of the PC group inoculated with tyramine-producing L. brevis PK11, isolated from a kimchi variety, was the highest among all the groups, exceeding the harmful level $(100 \mathrm{mg} / \mathrm{kg})$. This means that food safety risks associated with BAs (particularly tyramine) in kimchi will be greater when prolific tyramine-producing LAB strains such as L. brevis PK11 are the dominant indigenous strains. Therefore, starter or protective cultures should be developed to reduce the BA content in kimchi. Meanwhile, certain LAB, such as L. brevis PK11, with BA degradation capability are also able to largely produce BAs, leading to increased BA content in fermented kimchi. This suggests that when selecting possible $\mathrm{LAB}$ starter and/or protective cultures, both $\mathrm{BA}$ degradation and BA production must be considered in order to ensure the reduction in BA content in kimchi products.

Considering these selecting criteria, the present study found that the five LAB strains isolated from different kimchi varieties that were capable of degrading both toxic BAs (except for one strain) and incapable of producing both toxic BAs in vitro also had the capability to degrade the toxic BAs in Baechu kimchi fermentation experiments. Through the Baech $u$ kimchi fermentation, it was observed that L. brevis PK08 considerably reduced all six BAs, including polyamines as well as toxic BAs. Thus, the highest tyramine reduction compared to both the $\mathrm{C}$ group and PC group was seen in the LB group inoculated with L. brevis PK08, relative to the other inoculated groups, at rates of $66.65 \%$ and $81.89 \%$, respectively, while relatively high histamine, putrescine, cadaverine, spermidine, and spermine reductions were also seen. In addition, a gene that codes a BA-degrading enzyme, MCO, was detected in L. brevis PK08, which means that MCO is (at least in part) responsible for the BA reduction. The other $L A B$ strains showed similar or less BA reduction in Baechu kimchi than L. brevis PK08. Meanwhile, the optimal fermentation of each group was observed at different fermentation periods depending on the species and/or strains, because their distinct growth affected the $\mathrm{pH}$ and acidity of Baechu kimchi differently, thus determining the level of ripeness. Taken together, this study suggests that 
the LAB strains are valuable as starter or protective cultures in kimchi fermentation to reduce $B A$ content and prevent $B A-r e l a t e d$ food safety issues, and through the control of kimchi fermentation by the use of selected LAB strains, favorable levels of ripeness can also be achieved.

Supplementary Materials: The following are available online at https: / www.mdpi.com/article / 10.3390/microorganisms9122570/s1, Figure S1: Changes in BA content in Baechu kimchi inoculated with other selected LAB strains during fermentation, Table S1: Histamine and tyramine degradation by LAB strains previously isolated from kimchi varieties, Table S2: Histamine and tyramine degradation by LAB strains isolated from kimchi varieties in this study, Table S3: Changes in BA content during fermentation of Baechu kimchi inoculated with different LAB strains capable of degrading toxic BAs.

Author Contributions: Conceptualization, J.-H.M.; formal analysis, J.L.; investigation, J.L, Y.H.J. and A.M.P.; writing—original draft preparation, J.L.; writing—review and editing, J.L, Y.H.J., A.M.P. and J.-H.M.; supervision, J.-H.M. All authors have read and agreed to the published version of the manuscript.

Funding: This research was supported by the National Research Foundation of Korea (NRF) grants funded by the Korea Government (MSIT) (no. 2019R1H1A2100972 and no. 2020R1I1A3052118). Y.H.J. was supported by the Brain Korea 21 Fostering Outstanding Universities for Research from the Korean Ministry of Education. A.M.P. was supported by the Korea Government Scholarship Program (KGSP) from the National Institute for International Education (NIIED).

Acknowledgments: The authors thank Jong Hyoung Hong and Dabin Kim of the Department of Food and Biotechnology at Korea University for technical assistance.

Conflicts of Interest: The authors declare no conflict of interest.

\section{References}

1. Silla Santos, M.H. Biogenic amines: Their importance in foods. Int. J. Food Microbiol. 1996, 29, 213-231. [CrossRef]

2. Ladero, V.; Calles-Enríquez, M.; Fernández, M.; Alvarez, M.A. Toxicological effects of dietary biogenic amines. Curr. Nutr. Food Sci. 2010, 6, 145-156. [CrossRef]

3. European Food Safety Authority (EFSA). Scientific opinion on risk based control of biogenic amine formation in fermented foods. EFSA J. 2011, 9, 2393-2486. [CrossRef]

4. Smith, T.A. Amines in food. Food Chem. 1981, 6, 169-200. [CrossRef]

5. Taylor, S.L.; Eitenmiller, R.R. Histamine food poisoning: Toxicology and clinical aspects. CRC Crit. Rev. Toxicol. 1986, 17, 91-128. [CrossRef] [PubMed]

6. Bardócz, S. Polyamines in food and their consequences for food quality and human health. Trends Food Sci. Technol. 1995, 6 , 341-346. [CrossRef]

7. Warthesen, J.J.; Scanlan, R.A.; Bills, D.D.; Libbey, L.M. Formation of heterocyclic $N$-nitrosamines from the reaction of nitrite and selected primary diamines and amino acids. J. Agric. Food Chem. 1975, 23, 898-902. [CrossRef]

8. Ten Brink, B.; Damink, C.; Joosten, H.M.L.J.; Huis in 't Veld, J.H.J. Occurrence and formation of biologically active amines in foods. Int. J. Food Microbiol. 1990, 11, 73-84. [CrossRef]

9. Del Rio, B.; Redruello, B.; Linares, D.M.; Ladero, V.; Fernandez, M.; Martin, M.C.; Ruas-Madiedo, P.; Alvarez, M.A. The dietary biogenic amines tyramine and histamine show synergistic toxicity towards intestinal cells in culture. Food Chem. 2017, 218, 249-255. [CrossRef]

10. Del Rio, B.; Redruello, B.; Linares, D.M.; Ladero, V.; Ruas-Madiedo, P.; Fernandez, M.; Martin, M.C.; Alvarez, M.A. Spermine and spermidine are cytotoxic towards intestinal cell cultures, but are they a health hazard at concentrations found in foods? Food Chem. 2018, 269, 321-326. [CrossRef]

11. Del Rio, B.; Redruello, B.; Linares, D.M.; Ladero, V.; Ruas-Madiedo, P.; Fernandez, M.; Martin, M.C.; Alvarez, M.A. The biogenic amines putrescine and cadaverine show in vitro cytotoxicity at concentrations that can be found in foods. Sci. Rep. 2019, 9, 120. [CrossRef] [PubMed]

12. Del Rio, B.; Redruello, B.; Fernandez, M.; Cruz Martin, M.; Ladero, V.; Alvarez, M.A. The biogenic amine tryptamine, unlike $\beta$-phenylethylamine, shows in vitro cytotoxicity at concentrations that have been found in foods. Food Chem. 2020, 331, 127303. [CrossRef] [PubMed]

13. Linares, D.M.; Del Rio, B.; Redruello, B.; Ladero, V.; Martin, M.C.; Fernandez, M.; Ruas-Madiedo, P.; Alvarez, M.A. Comparative analysis of the in vitro cytotoxicity of the dietary biogenic amines tyramine and histamine. Food Chem. 2016, 197, 658-663. [CrossRef] 
14. European Commission (EC). Commission Regulation No. 2073/2005 of 15th November 2005 on microbiological criteria for foodstuffs. Off. J. Eur. Union 2005, L338, 1-25.

15. U.S. Food and Drug Administration (FDA). Fish and Fishery Products Hazards and Controls Guidance, 4th ed.; Center for Food Safety and Applied Nutrition: Rockville, MD, USA, 2011.

16. Cheigh, H.-S.; Park, K.-Y.; Lee, C.Y. Biochemical, microbiological, and nutritional aspects of kimchi (Korean fermented vegetable products). Crit. Rev. Food Sci. Nutr. 1994, 34, 175-203. [CrossRef]

17. Codex Alimentarius Commission. Codex Standard for Kimchi; Codex Stan 223-2001; Food and Agriculture Organization of the United Nations: Rome, Italy, 2001.

18. Jung, M.Y.; Kim, T.-W.; Lee, C.; Kim, J.Y.; Song, H.S.; Kim, Y.B.; Ahn, S.W.; Kim, J.S.; Roh, S.W.; Lee, S.H. Role of jeotgal, a Korean traditional fermented fish sauce, in microbial dynamics and metabolite profiles during kimchi fermentation. Food Chem. 2018, 265, 135-143. [CrossRef] [PubMed]

19. Cho, J.; Lee, D.; Yang, C.; Jeon, J.; Kim, J.; Han, H. Microbial population dynamics of kimchi, a fermented cabbage product. FEMS Microbiol. Lett. 2006, 257, 262-267. [CrossRef]

20. Jung, J.Y.; Lee, S.H.; Jin, H.M.; Hahn, Y.; Madsen, E.L.; Jeon, C.O. Metatranscriptomic analysis of lactic acid bacterial gene expression during kimchi fermentation. Int. J. Food Microbiol. 2013, 163, 171-179. [CrossRef]

21. Jeong, S.H.; Lee, S.H.; Jung, J.Y.; Choi, E.J.; Jeon, C.O. Microbial succession and metabolite changes during long-term storage of kimchi. J. Food Sci. 2013, 78, M763-M769. [CrossRef]

22. Jung, J.Y.; Lee, S.H.; Jeon, C.O. Kimchi microflora: History, current status, and perspectives for industrial kimchi production. Appl. Microbiol. Biotechnol. 2014, 98, 2385-2393. [CrossRef]

23. Hwang, S.-Y.; Hur, Y.-M.; Choi, Y.-H.; Rhee, S.-H.; Park, K.-Y.; Lee, W.-H. Inhibitory effect of kimchi extracts on mutagenesis of aflatoxin $\mathrm{B}_{1}$. Environ. Mutagens Carcinog. 1997, 17, 133-137.

24. Islam, M.S.; Choi, H. Antidiabetic effect of Korean traditional Baechu (Chinese cabbage) kimchi in a type 2 diabetes model of rats. J. Med. Food 2009, 12, 292-297. [CrossRef] [PubMed]

25. Kim, E.K.; An, S.-Y.; Lee, M.-S.; Kim, T.H.; Lee, H.-K.; Hwang, W.S.; Choe, S.J.; Kim, T.-Y.; Han, S.J.; Kim, H.J.; et al. Fermented kimchi reduces body weight and improves metabolic parameters in overweight and obese patients. Nutr. Res. 2011, 31, 436-443. [CrossRef]

26. Kim, B.; Park, K.Y.; Kim, H.Y.; Ahn, S.C.; Cho, E.J. Anti-aging effects and mechanisms of kimchi during fermentation under stress-induced premature senescence cellular system. Food Sci. Biotechnol. 2011, 20, 643-649. [CrossRef]

27. Kim, B.K.; Choi, J.M.; Kang, S.A.; Park, K.Y.; Cho, E.J. Antioxidative effects of Kimchi under different fermentation stage on radical-induced oxidative stress. Nutr. Res. Pract. 2014, 8, 638-643. [CrossRef]

28. Kim, H.-Y.; Song, J.-L.; Chang, H.-K.; Kang, S.-A.; Park, K.-Y. Kimchi protects against azoxymethane/dextran sulfate sodiuminduced colorectal carcinogenesis in mice. J. Med. Food 2014, 17, 833-841. [CrossRef] [PubMed]

29. Mah, J.-H.; Kim, Y.J.; No, H.-K.; Hwang, H.-J. Determination of biogenic amines in kimchi, Korean traditional fermented vegetable products. Food Sci. Biotechnol. 2004, 13, 826-829.

30. Park, Y.K.; Lee, J.H.; Mah, J.-H. Occurrence and reduction of biogenic amines in kimchi and Korean fermented seafood products. Foods 2019, 8, 547. [CrossRef]

31. Cho, T.-Y.; Han, G.-H.; Bahn, K.-N.; Son, Y.-W.; Jang, M.-R.; Lee, C.-H.; Kim, S.-H.; Kim, D.-B.; Kim, S.-B. Evaluation of biogenic amines in Korean commercial fermented foods. Korean J. Food Sci. Technol. 2006, 38, 730-737.

32. Jin, Y.H.; Lee, J.H.; Park, Y.K.; Lee, J.-H.; Mah, J.-H. The occurrence of biogenic amines and determination of biogenic amineproducing lactic acid bacteria in Kkakdugi and Chonggak kimchi. Foods 2019, 8, 73. [CrossRef]

33. Kang, K.H.; Kim, S.H.; Kim, S.-H.; Kim, J.G.; Sung, N.-J.; Lim, H.; Chung, M.J. Analysis and risk assessment of Nnitrosodimethylamine and its precursor concentrations in Korean commercial kimchi. J. Korean Soc. Food Sci. Nutr. 2017, 46, 244-250. [CrossRef]

34. Lee, J.-H.; Jin, Y.H.; Park, Y.K.; Yun, S.J.; Mah, J.-H. Formation of biogenic amines in Pa (green onion) kimchi and Gat (mustard leaf) kimchi. Foods 2019, 8, 109. [CrossRef]

35. Tsai, Y.-H.; Kung, H.-F.; Lin, Q.-L.; Hwang, J.-H.; Cheng, S.-H.; Wei, C.-I.; Hwang, D.-F. Occurrence of histamine and histamineforming bacteria in kimchi products in Taiwan. Food Chem. 2005, 90, 635-641. [CrossRef]

36. Gardini, F.; Özogul, Y.; Suzzi, G.; Tabanelli, G.; Özogul, F. Technological factors affecting biogenic amine content in foods: A review. Front. Microbiol. 2016, 7, 1218. [CrossRef]

37. Mah, J.-H.; Park, Y.K.; Jin, Y.H.; Lee, J.-H.; Hwang, H.J. Bacterial production and control of biogenic amines in Asian fermented soybean foods. Foods 2019, 8, 85. [CrossRef]

38. Callejón, S.; Sendra, R.; Ferrer, S.; Pardo, I. Identification of a novel enzymatic activity from lactic acid bacteria able to degrade biogenic amines in wine. Appl. Microbiol. Biotechnol. 2012, 98, 185-198. [CrossRef]

39. Dapkevicius, M.L.E.; Nout, M.R.; Rombouts, F.M.; Houben, J.H.; Wymenga, W. Biogenic amine formation and degradation by potential fish silage starter microorganisms. Int. J. Food Microbiol. 2000, 57, 107-114. [CrossRef]

40. Tosukhowong, A.; Visessanguan, W.; Pumpuang, L.; Tepkasikul, P.; Panya, A.; Valyasevi, R. Biogenic amine formation in Nham, a Thai fermented sausage, and the reduction by commercial starter culture, Lactobacillus plantarum BCC 9546. Food Chem. 2011, 129, 846-853. [CrossRef] [PubMed] 
41. Guarcello, R.; De Angelis, M.; Settanni, L.; Formiglio, S.; Gaglio, R.; Minervini, F.; Moschetti, G.; Gobbetti, M. Selection of amine-oxidizing dairy lactic acid bacteria and identification of the enzyme and gene involved in the decrease of biogenic amines. Appl. Environ. Microbiol. 2016, 82, 6870-6880. [CrossRef]

42. Li, L.; Wen, X.; Wen, Z.; Chen, S.; Wang, L.; Wei, X. Evaluation of the biogenic amines formation and degradation abilities of Lactobacillus curvatus from Chinese bacon. Front. Microbiol. 2018, 9, 1015. [CrossRef]

43. Leuschner, R.G.; Heidel, M.; Hammes, W.P. Histamine and tyramine degradation by food fermenting microorganisms. Int. J. Food Microbiol. 1998, 39, 1-10. [CrossRef]

44. Kim, S.-H.; Kim, S.H.; Kang, K.H.; Lee, S.; Kim, S.J.; Kim, J.G.; Chung, M.J. Kimchi probiotic bacteria contribute to reduced amounts of N-nitrosodimethylamine in lactic acid bacteria-fortified kimchi. LWT Food Sci. Technol. 2017, 84, 196-203. [CrossRef]

45. Jeong, S.H.; Lee, H.J.; Jung, J.Y.; Lee, S.H.; Seo, H.-Y.; Park, W.-S.; Jeon, C.O. Effects of red pepper powder on microbial communities and metabolites during kimchi fermentation. Int. J. Food Microbiol. 2013, 160, 252-259. [CrossRef] [PubMed]

46. AOAC. Official Methods of Analysis of AOAC International, 18th ed.; AOAC International: Gaithersburg, MD, USA, 2005.

47. Yoon, H.; Park, J.H.; Choi, A.; Hwang, H.-J.; Mah, J.-H. Validation of an HPLC analytical method for determination of biogenic amines in agricultural products and monitoring of biogenic amines in Korean fermented agricultural products. Toxicol. Res. 2015, 31, 299-305. [CrossRef]

48. NCBI. Primer-BLAST. Available online: https:/ / www.ncbi.nlm.nih.gov/tools/primer-blast/ (accessed on 17 November 2021).

49. NCBI. BLAST. Available online: http:/ / www.ncbi.nlm.nih.gov/BLAST/ (accessed on 17 November 2021).

50. Kim, M.-J.; Kim, K.-S. Tyramine production among lactic acid bacteria and other species isolated from kimchi. LWT Food Sci. Technol. 2014, 56, 406-413. [CrossRef]

51. Lee, C.-H. Lactic acid fermented foods and their benefits in Asia. Food Control 1997, 8, 259-269. [CrossRef]

52. Alvarez, M.A.; Moreno-Arribas, M.V. The problem of biogenic amines in fermented foods and the use of potential biogenic amine-degrading microorganisms as a solution. Trends Food Sci. Technol. 2014, 39, 146-155. [CrossRef]

53. Herrero-Fresno, A.; Martínez, N.; Sánchez-Llana, E.; Díaz, M.; Fernández, M.; Martin, M.C.; Ladero, V.; Alvarez, M.A. Lactobacillus casei strains isolated from cheese reduce biogenic amine accumulation in an experimental model. Int. J. Food Microbiol. 2012, 157, 297-304. [CrossRef]

54. Mheen, T.-I.; Kwon, T.-W. Effect of temperature and salt concentration on kimchi fermentation. Korean J. Food Sci. Technol. 1984, $16,443-450$.

55. Dierick, N.; Vandekerckhove, P.; Demeyer, O. Changes in nonprotein nitrogen compounds during dry sausage ripening. J. Food Sci. 1974, 39, 301-304. [CrossRef]

56. Barbieri, F.; Montanari, C.; Gardini, F.; Tabanelli, G. Biogenic amine production by lactic acid bacteria: A review. Foods 2019, 8, 17. [CrossRef]

57. Bonnin-Jusserand, M.; Grandvalet, C.; Rieu, A.; Weidmann, S.; Alexandre, H. Tyrosine-containing peptides are precursors of tyramine produced by Lactobacillus plantarum strain IR BL0076 isolated from wine. BMC Microbiol. 2012, 12, 199. [CrossRef]

58. Costantini, A.; Pietroniro, R.; Doria, F.; Pessione, E.; Garcia-Moruno, E. Putrescine production from different amino acid precursors by lactic acid bacteria from wine and cider. Int. J. Food Microbiol. 2013, 165, 11-17. [CrossRef] [PubMed]

59. Ladero, V.; Cañedo, E.; Pérez, M.; Martín, M.C.; Fernández, M.; Alvarez, M.A. Multiplex qPCR for the detection and quantification of putrescine-producing lactic acid bacteria in dairy products. Food Control 2012, 27, 307-313. [CrossRef]

60. Poveda, J.M.; Ruiz, P.; Sesena, S.; Palop, M.L. Occurrence of biogenic amine-forming lactic acid bacteria during a craft brewing process. LWT Food Sci. Technol. 2017, 85, 129-136. [CrossRef]

61. Shin, S.-W.; Kim, Y.-S.; Kim, Y.-H.; Kim, H.-T.; Eum, K.-S.; Hong, S.-R.; Kang, H.-J.; Park, K.-H.; Yoon, M.-H. Biogenic-amine contents of Korean commercial salted fishes and cabbage kimchi. Korean J. Fish. Aquat. Sci. 2019, 52, $13-18$.

62. Jin, H.S.; Kim, J.B.; Yun, Y.J.; Lee, K.J. Selection of kimchi starters based on the microbial composition of kimchi and their effects. J. Korean Soc. Food Sci. Nutr. 2008, 37, 671-675. [CrossRef]

63. Kim, S.J. Field difficulties in modernization of kimchi industry. Food Ind. Nutr. 2001, 6, 34-37.

64. Pištěková, H.; Jančová, P.; Berčíková, L.; Buňka, F.; Sokolová, I.; Šopík, T.; Maršálková, K.; de Amaral, O.M.R.P.; Buňková, L. Application of qPCR for multicopper oxidase gene (MCO) in biogenic amines degradation by Lactobacillus casei. Food Microbiol. 2020, 91, 103550. [CrossRef] [PubMed]

65. Sun, S.; Jiang, D.; Fan, M.; Li, H.; Jin, C.; Liu, W. Selection of a versatile Lactobacillus plantarum for wine production and identification and preliminary characterisation of a novel histamine-degrading enzyme. Int. J. Food Sci. Technol. 2020, 55, 2608-2618. [CrossRef] 